\title{
DESTRUCTIVE TUBERCULOSIS IN THE EYE OF A CHILD.
}

\author{
Hiram R. STILWILL, M.D., F.A.C.S.
}

DENVER, COLORADO.

\begin{abstract}
This case of obscure ocular disease destroyed the function of the eye and led to enucleation. The pathologic examinations demonstrated the tuberculous character of the lesions. Read before the Colorado Congress, July, 1921.
\end{abstract}

Acute tuberculosis occurring in the eye of a child so young (four years) is extremely rare. It is said to occur more often between the age of five and twenty-five, (de Schweinitz) the average age being twelve years.

The case reported began as an iritis or iridocyclitis, later involved the sclera and cornea, taking on a confluent or conglomerate form, and resulting in rapid destruction of the eye in a comparatively short period of time, some seven weeks from its onset.

CASE. David H. age 4 years, American, was first seen on June 6, 1921. The following history was obtained. His mother stated that she first noticed a redness of the right eye, about three weeks before, which she attributed to a "cold." There had been no injury to the eye and no secretion had been noticed. He had not complained of pain or discomfort, and had not been ill. He is the youngest of four boys, and has always been healthy; has had no illness. The family history is negative. Both parents living and well and no history of $T$. B. on either side is obtainable. Physical examination by the family physician was reported negative; and no enlarged tonsils or cervical glands were noted.

Examination revealed a moderately congested right eyeball, more marked in the ciliary zone. The cornea was clear, anterior chamber deep and the aqueous somewhat cloudy. The iris appeared dark brown in color (the left iris being light blue). The pupil was contracted, fixed and irregular, with a grayish white exudate in the pupillary area. The tension was normal and there was no bulbar tenderness. Vision was reduced to hand movements. The left eye was normal.

On June 8th Dr. Ward Burdick, who examined the blood, reported a negative Wassermann. The spinal fluid was not taken. The von Pirquet skin test for T. B. was pronounced "mildly positive." Instillations of atropin had no effect whatsoever on the adhesions.

The patient was seen frequently during the succeeding ten days, during which time several small grayish white deposits appeared in the stroma of the iris near the pupillary margin, and small blood vessels were seen running thru it. The anterior chamber became more shallow, the cornea infiltrated and uniformly hazy without vascularity. At the end of three weeks the sclera near the limbus on the nasal side became bluish in color, and an ectasia 3 to $4 \mathrm{~mm}$. in width involving the nasal half of the sclera presented. The tension was now recorded as minus. During this time the patient did not complain of pain or discomfort and except for a slight languor was apparently quite well.

As the eye was rapidly becoming destroyed, its removal was strongly advised, but consent was not obtained until July 6th, when enucleation was done.

Report submitted by Dr. Philip Hillkowitz, Pathologist at Mercy Hospital:

Naked eye appearance. "Right eye, ectasia at limbus involving inner half of globe."

"Microscopic examination: "Sections thru iris show on either side of the stratum pigmentosum a band of tissue, in which are seen many tubercles with characteristic giant cells of the Langhans' type, epithelioid cells and small round cells, as well as foci of caseation necrosis. Diagnosis: Tuberculosis."

Report of Dr. Wm. C. Finnoff:

After removal, the eye was fixed in formalin solution.

Macroscopic appearance: The submitted specimen had been cut meridionally and a large $\mathrm{V}$-shaped piece of cornea removed. The remaining cornea was pushed forward by a collar like ectasia 
in the region of the limbus. This portion of the eye was markedly thickened. A yellowish white exudate rested on the anterior surface of the iris, and almost completely filled the anterior chamber. The lens was not present. The retina was detached from the choroid. This probably occurred during fixation and opening of the globe.

Time did not permit of proper embedding of the globe, and only half of the eye was sectioned. This corresponded to the portion from which the cornea had been removed to prepare frozen sections. Celloidin embedding and staining with hematoxylin and eosin.

Microscopic findings: The anterior portion of the section, corresponding to the region just posterior to the limbus, is made up of a mass of lymphocytes, endothelial cells, giant cells of the Langhans' type, serous exudate, and necrotic material. This is covered externally by a thin layer of subconjunctival tissue, which contains conjunctival vessels. The mass is $3 \mathrm{~mm}$. in thickness. In this area, the sclera has been replaced by a granulomatous mass, and no trace of scleral bundles can be found. The circular and radial muscle bundles of the ciliary body are entirely destroyed, and only phantom processes of the ciliary body are seen in the center of the mass. These consist of a wavy line of pigment granules, which are left in the wake of pigment epithelium, which has degenerated. Internal to the line of pigment granules, a cyclitic membrane has invaded the vitreous. This mass is typically granulomatous, and con- tains giant cells and necrotic material. The cyclitic mass extends backward, almost to the ora serrata. A thin layer of broken down scleral bundles, corresponding to the external layer, is first noted opposite the posterior $1 / 4$ of the corona ciliaris. Here the bundles are separated by cellular exudate and giant cells. Opposite the beginning of the orbicular ciliaris, the sclera is of a normal thickness and is not involved. Ciliary epithelial cells are first seen opposite the posterior $1 / 4$ of the corona ciliaris. At the ora serrata, the retina is invaded by a few small round cells. The vitreous in this region contains fibrin and scattered endothelial cells. The endothelial cells are gathered in clumps, varying from 2 to 5 or 6 cells in thickness, on the inner surface of the retina, in the region of the peripheral retinal blood vessels. The nerve fiber and ganglion cell layers of the retina are slightly edematous. The retina was partially detached antemortem, as is indicated by a thin layer of fibrin and newly proliferated epithelial cells, which contain a few pigment granules. The layers of the choroid are widely separated by a serous exudate in its anterior half. This is especially marked in the outer portion, where the suprachoroidal space is distended to a marked degree. The optic nerve, cornea, and iris are not included in the sections cut.

Time did not permit staining for tubercle bacilli.

Diagnosis: Tuberculosis of the ciliarv body, with extension thru the globe. 